\title{
Microemulsions: a novel approach to enhanced oil recovery: a review
}

\author{
Achinta Bera $\cdot$ Ajay Mandal
}

Received: 19 March 2014 / Accepted: 15 September 2014/Published online: 24 September 2014

(C) The Author(s) 2014. This article is published with open access at Springerlink.com

\begin{abstract}
The trend of growing interest in alternative source of energy focuses on renewable products worldwide. However, the situation of petroleum industries in many countries needs much concern in improving the oil recovery technique. Chemical method, especially microemulsion flooding, plays an important role in enhanced oil recovery technique due to its ability to reduce interfacial tension between oil and water to a large extent as well as alter wettability of reservoir rocks. Surfactant-based chemical systems have been reported in many academic studies and their technological implementations are potential candidates in enhanced oil recovery activities. This paper reviews the role of different types of surfactants in enhanced oil recovery, structure of microemulsion, phase behavior of oil-brine-surfactant/cosurfactant systems with variation of different parameters such as salinity, temperature, pressure and physicochemical properties of microemulsions including solubilization capacity, interfacial tension, viscosity and density under reservoir conditions. The enhanced oil productivity by microemulsion flooding with different surfactant/cosurfactant systems has also been discussed in this paper. This review introduces a new opening in enhanced oil recovery by microemulsion flooding with some new aspects.
\end{abstract}

\footnotetext{
A. Bera $(\bowtie)$

Department of Civil and Environmental Engineering, School of Mining and Petroleum Engineering, University of Alberta, Edmonton, AB T6G 2W2, Canada

e-mail: achintachm@gmail.com

A. Mandal

Department of Petroleum Engineering, Indian School of Mines, Dhanbad 826004, India
}

Keywords Microemulsion - Enhanced oil recovery · Interfacial tension $\cdot$ Phase behavior $\cdot$ Solubilization capacity $\cdot$ Microemulsion flooding

\section{Introduction}

The energy demand will be met by a global energy mix that is undergoing a transition from the current dominance of fossil fuels to a more balanced distribution of energy sources. New discoveries of conventional oil fields are declining, while demand for oil is increasing day by day, particularly in the developed and developing countries. After conventional waterflood processes, the residual oil in the reservoir remains as a discontinuous phase in the form of oil drops trapped by capillary forces and is likely to be around $70 \%$ of the original oil in place (OOIP) (Dosher and Wise 1976). However, technically it is possible to improve this recovery efficiency by applying enhanced oil recovery (EOR) processes.

Microemulsion is an efficient tool in EOR techniques because of its high level of extraction efficiency by reducing oil-water interfacial tension (Santanna et al. 2009; Bera et al. 2014a). Microemulsions are transparent and translucent homogeneous mixtures of hydrocarbons and water with large amounts of surfactants (Schulman et al. 1959; Stoeckenius et al. 1960). Alkanols (medium chain alcohols such as propanol, butanol, isoamyl alcohol, pentanol, hexanol, etc.) are generally used as cosurfactants for the preparation of microemulsions (Barakat et al. 1983; Lalanne-Cassou et al. 1983). The solubility of alkanol in water depends on the alcohol chain length. Short-chain alcohols such as methanol and ethanol are able to undergo a miscibility process with water. On the other hand, medium or long-chain alcohols (from propanol to higher alcohol) show very low solubility in water.

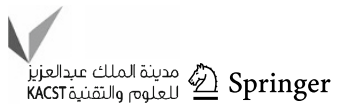


In recent years, microemulsion flooding has become immensely important in the petroleum industries for the EOR technique (Santanna et al. 2009; Southwick et al. 2010; Kumar and Mohanty 2010; Flaaten et al. 2010; Elraies et al. 2010; Jeirani et al. 2013a, b; Bera et al. 2014b). Various research projects are involved in this field aiming to improve the petroleum oil recovery from natural oil reservoirs. The oil recovery process can be divided into mainly three stages such as primary recovery, secondary recovery and tertiary recovery (Gurgel et al. 2008). In primary oil recovery, oil is recovered due to pressure maintenance, in which oil is forced out through the production well by natural forces and reservoir gravity. The intrinsic or natural capacity of oil fields for producing oil is, however, promoted via primary recovery techniques. But physical constraints such as reduced well pressure and extensive oil trapping lessen oil production which eventually ceased at one stage and caused changes in the composition of crude oil affecting the reservoir wettability (Yangming et al. 2003). Chilingar and Yen (1983) thoroughly investigated different reservoir cores such as limestone, dolomite limestone, calcite dolomite and dolomite and concluded that $15 \%$ were strongly oil wet, $65 \%$ were oil wet, $12 \%$ moderate oil wet and $8 \%$ water wet. Therefore, in this point of view, it is an important issue regarding wettability for further desired oil recovery. When the required pressure is not available to expel the oil, water is injected to create pressure to recover the oil. This is generally called secondary oil recovery or water flooding. The primary and secondary oil recovery process can recover nearly $30-35 \%$ of OOIP. To recover the remaining oil, a tertiary recovery process is used. This tertiary oil recovery is also known as enhanced oil recovery. Tertiary EOR technique can be divided into mainly three categories, viz. chemical flooding, thermal process and gas injection. Alkali flooding, polymer flooding and micellar-polymer flooding are examples of chemical flooding. On the other hand, in situ combustion, steam injection and wet combustion methods are grouped into thermal processes (Leung et al. 1985; Sharma and Shah 1985; Auvray et al. 1984; Scriven 1976). Over the years, a number of innovative EOR processes such as microbial enhanced oil recovery (MEOR) and ultrasonic vibration methods have been introduced noticeably. Another important technique is foam flooding. Foam is used during gas flooding such as with steam, $\mathrm{CO}_{2}$ and miscible gas for mobility control. Sometimes, steam foams are used extensively to improve vertical and areal sweep efficiency and to reduce steam channeling in a shallow heavy oil reservoir. The steam foam may consist of surfactant with or without noncondensable gas. Steam foams have been used in conjunction with both continuous and cyclic steam injection. The classifications of EOR methods are shown in Fig. 1.

Capillary forces are also the important parameters for recovery of residual oil. These capillary forces are normally quantified by Young-Laplace equations in interfacial sciences (Schramm et al. 2003). The phase behavior of surfactant/cosurfactant-brine-oil system is the key factor in interpreting the performance of oil recovery by the microemulsion flooding process. Due to the well-established relationship between the microemulsion phase behavior and interfacial tension (IFT), it is common in the industry to screen surfactants and their formulations for low IFT through oil-water phase behavior tests (Shah 1981; Levitt et al. 2006; Engelskirchen et al. 2007; Kayalia et al. 2010). The development of microemulsions for specific oilfield applications requires a systematic study of phase behavior as an important tool to select a treatment composition that satisfies specific parameters defined by the application. To identify microemulsion phase boundary, it is very common to study phase behavior in the laboratory. The formation and stability of microemulsion systems are driven by very low water-oil interfacial tension to compensate the large increase in the dispersion entropy (Bumajdad and Eastoe 2004). In the past several years, it has been shown that the phase behavior of surfactant/cosurfactant-oil-brine/water system is of the intense importance in the interpretation and forecasting the scopes of applications in the field of EOR techniques (Shah and Schechter 1977). At present, it is common that formulation of surfactant/cosurfactant-brineoil systems that exhibit desirable phase behavior is an important stage in optimizing the performance of microemulsion systems for EOR methods (Healy and Reed 1974; Healy et al. 1975; Bera et al. 2012a). In surfactant/cosurfactant-oil-brine systems, microemulsion shows different phase behavior with variation of different parameters such as salinity, temperature and pressure. The commonly observed Winsor-type system (Winsor 1954; Abe et al. 1987; Nakamae et al. 1990; Bera et al. 2012b) indicates that the microemulsions can exist in equilibrium with excess oil, excess water or both. In a Winsor-type I system, lower phase microemulsion exists with excess oil, and in case of Winsor-type II, upper phase microemulsion exists with excess brine. In general, middle-phase microemulsion (Healy et al. 1976) (surfactant-rich middle phase) system is known as Winsor-type III microemulsion and has a bicontinuous structure made by an equal mixture of water-inoil and oil-in-water type of microemulsions (Schulman et al. 1959; Stoeckenius et al. 1960). The single-phase microemulsion region is called Winsor-type IV phase. To represent these four phases, another notation system, especially employed by Kahlweit et al. (1990), uses the symbols $\underline{2}, \overline{2}, 3$ and 1 , respectively. The factors that affect 
Fig. 1 Simplified classification of EOR methods

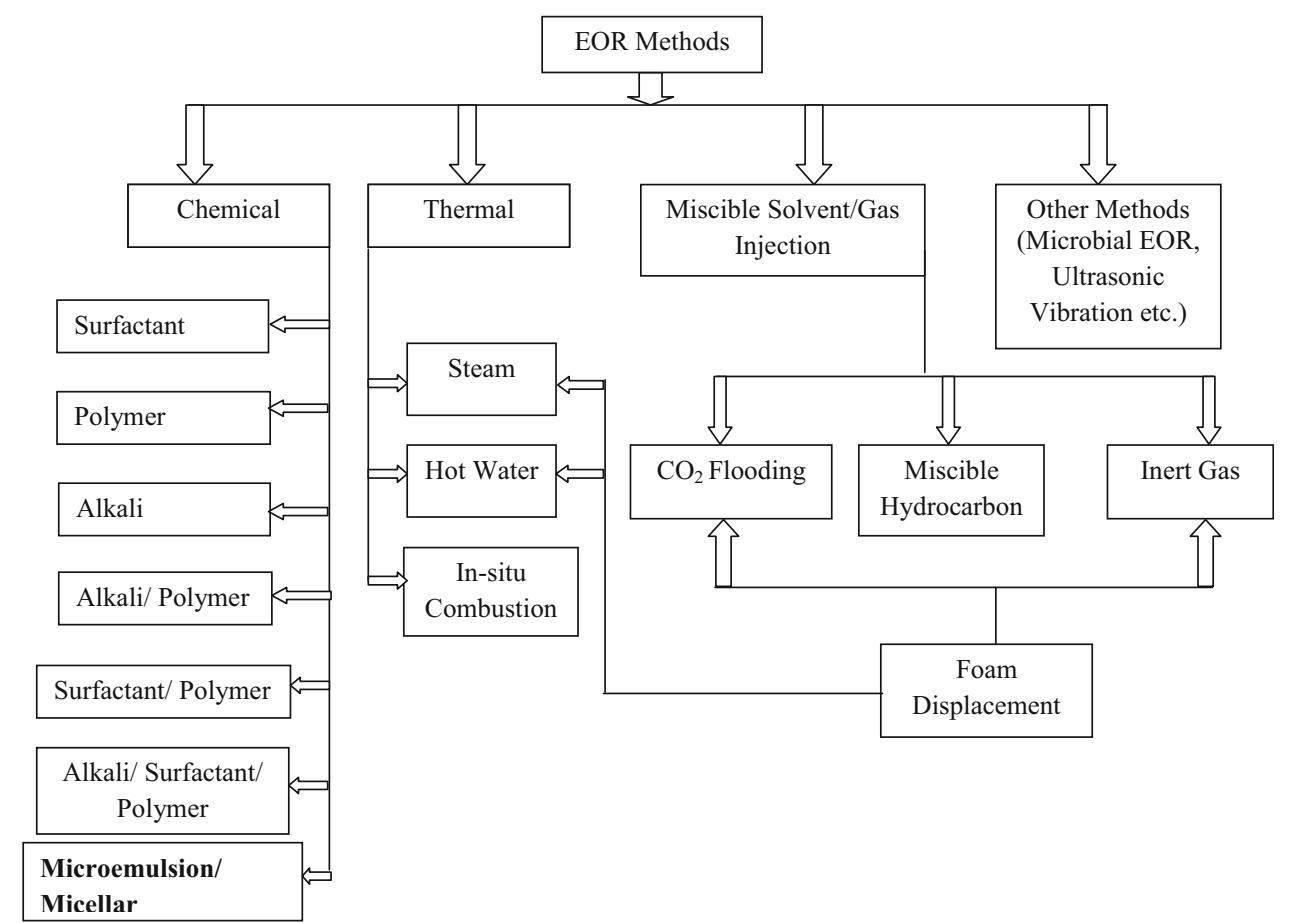

the phase transition between different types of systems include the salinity, temperature, molecular structure and nature of the surfactant and cosurfactant and the nature of the oil and water-oil ratio (WOR) (Shah 1985).

In this review paper, the phase behavior of surfactant/ cosurfactant-oil-brine system and the factors that affect the phase behavior and interfacial tension of the systems have been discussed. This paper also reviews the role of interfacial tension in oil recovery and its relation with phase behavior. In this connection, other properties of microemulsions (such as solubilization capacity, density and viscosity) that are directly or indirectly related with oil recovery have been also discussed in this review paper.

\section{Surfactants in EOR Techniques}

The choice of surfactants for EOR is another factor for efficient extraction of trapped oil from oil reservoirs. Surfactant solutions for use in EOR can have high $(2.0-10.0 \mathrm{wt} \%)$ or low $(0.1-0.2 \mathrm{wt} \%)$ surfactant concentration. To lower the interfacial tension up to an ultralow value, low surfactant concentration system was used where the aqueous phase of the surfactant solution is about the apparent critical micelle concentration (CMC). In case of high surfactant concentration systems, a middle-phase microemulsion is formed that is in equilibrium with excess oil and excess brine. The basic components of this microemulsion are surfactant, water, oil, alcohol and salts (generally, $\mathrm{NaCl}$ is used). High surfactant concentrations in the injected plug result in a relatively small pore volume (about 3-20\%) compared to micellar solutions (15-60\%). Different surfactants were used to verify their activities in EOR techniques by laboratory experiments. Screening of surfactants for EOR in laboratory is done based on the phase behavior experiments. Screening of petroleum sulfonates, which are generally known as the most available, commercial types of surfactants and manipulation of their combination in a chemical slug have been always of particular interest to researchers. In 1984, Bostich et al. (1984) invented an aqueous petroleum sulfonate mixture, which contained, at least, two different petroleum sulfonates. The implemented sulfonates had an average molecular weight within the range of about $300-450 \mathrm{~g} / \mathrm{mol}$. The hydrocarbon portion of the sulfonate had an average aliphatic to aromatic portion ratio within the range of about 4-20 mol per mole. In microemulsion flooding, anionic surfactants were used extensively. Several surfactants have been used in laboratory experiments for their screening for use in large scale. Few of the surfactants and their activities are discussed in Table 1.

\section{Microemulsions in EOR}

\section{Compositions of microemulsions}

It is well known that microemulsions are generally composed of hydrocarbons, surfactants/cosurfactants and brine. Surfactants are considered to be the principal constituents 
Table 1 Surfactants used in laboratory studies in enhanced oil recovery

\begin{tabular}{lll}
\hline Sl. no. & Author(s) & Names of the surfactants and types \\
\hline 1. & Holm (1971) & Sodium sulfonates (anionic) \\
2. & Healy et al. (1975) & $\begin{array}{l}\text { Monoethanol amine salt of } \\
\text { alkylorthoxylene sulfonic acid (anionic) }\end{array}$
\end{tabular}

Brief descriptions

3. Healy and Reed (1977)

4. Glover et al. (1979)

5. Meyers and Salter (1980)

6. Willhite et al. (1980)

7. Verkruyse and Salter (1985)

8. Sayyouh et al. (1991)

9. Maerker and Gale (1992)

10. Purwono and Murachman (2001)

11. Bouabboune et al. (2006)

12. Chai et al. (2007)

13. Santanna et al. (2009)

14. Zhu et al. (2009)
Ethoxylated alcohols (nonionic)

Alkali surfactant NM (anioinic)

Nonyl surfactant (anionic)

Monoethanol amine salt of dodecylorthoxylene sulfonic acid (anionic)

A sulfonated petroleum derivative (anionic)

TRS $10-80$ petroleum sulfonate (anionic)

WITCO TRS-18 petroleum sulfonate (anionic)

A blend of two tridecyl alcohol sulfates (PL612 + P1613) (anionic)

Sodium ligno sulfonate (anionic)

Sodium dodecyl sulfonate, sodium dodecyl sulfate and sodium dodecyl benzene sulfonate (all are anionic)

Soap sodium soap and a synthetic surfactant (anionic)

Triton X100 (nonionic), cetyltrimethylammonium bromide (cationic)
It was concluded that a clear microemulsion can be formed over a wide range of temperature by using this surfactant, and the microemulsion showed efficient extraction efficiency at elevated temperature

They concluded that microemulsion flooding can be considered as a miscible process before breakdown of slug and then it will be immiscible. It was also stated that surfactant retention in porous media is a serious problem in microemulsion flooding

It was reported that ultralow interfacial tension between microemulsion-oil systems increases the oil recovery efficiency

Depending on the salinity surfactant retention varied from 0.16 to $0.65 \mathrm{mg} / \mathrm{g}$ and rock and phase behavior was affected by salinity; as salinity changes from low to high values, middle-phase to lower-phase inversion takes place

Dynamic adsorption study of the surfactant was reported in Berea sandstone at different oil/brine ratios

In these microemulsion displacements a new milky microemulsion was produced by mixing of the injected one and it followed the oil bank. The efficiency of the microemulsion was quite high. The adsorption of the surfactant on rock surface was also significant

The microemulsion made with this surfactant did not show the desired result of oil recovery, but reduced the interfacial tension and showed high solubilization capacity

With this surfactant, flooding experiments were carried out and satisfactory results were recorded. Phase behavior was also investigated with this microemulsion

This surfactant was used to prepare diesel oil microemulsion. This is a cost-effective formulation of microemulsion and gives good results in oil recovery experiments

The microemulsion formulated with the surfactant was useful, but the compositions are not available and so the use of this microemulsion is not always possible

With this surfactant, a comparative study was conducted between surfactant and microemulsion flooding. The result showed a higher efficiency of microemulsion than the surfactant solution alone

Phase behavior study of microemulsion was carried out with these surfactants. The formulated of the microemulsions showed different $\varepsilon-\beta$ phase behaviors

The microemulsions are very much efficient to recover residual, and viscosity of the microemulsion also plays an important role here. The more viscous microemulsion led to more oil recovery than the other one with lower viscosity

Triton X-100 and its oligomer tyloxapol with cetyltrimethylammonium bromide induced by hydrolyzed polyacrylamide was used to produce ultralow interfacial tension between crude oil and aqueous phase (brine). This combined mixture is very much effective for lowering interfacial tension and applicable for additional oil recovery 
Table 1 continued

\begin{tabular}{|c|c|c|}
\hline S1. no. & Author(s) & Names of the surfactants and types \\
\hline 15 . & Iglauer et al. (2010) & $\begin{array}{l}\text { Di-tridecyl sulfosuccinic acid ester } \\
\text { (Aerosol TR-70), coconut } \\
\text { diethanolamide (CW-100), } \\
\text { alkylpolyglycosides (Agrimul PG2062 } \\
\text { and Agrimul PG2069), alkylpropoxy } \\
\text { sulfate sodium salts (Alfoterra } 23 \text { and } \\
\text { Alfoterra 38) (all are anionic) }\end{array}$ \\
\hline
\end{tabular}

16. Wan et al. (2011)

17. Trabelsi et al. (2011)

18. Qiao et al. (2012)

19. Mandal et al. (2012)

20. Gao and Sharma (2013)

21. Lu et al. (2014)

22. McLendon et al. (2014)

23. Bai et al. (2014)
Span-80 and Tween 80 (nonionic)

Triton X405 (nonionic), sodium dodecyl sulfate (anionic), sodium dodecylbenzene sulfonate (anionic)

1,3,5-triazine surfactants (Nonionic)

Tergitol 15-S-5, Tergitol 12-S-7, Tergitol 15-S-9, Tergitol 15-S-12 (nonionic)

Alkyl sulfate gemini surfactant (anionic)

Guerbet alkoxy sulfate (GAS) surfactants, Guerbet alkoxy carboxylate surfactants (anionic)

Branched nonylphenol ethoxylates (Huntsman SURFONICs N-120 and Huntsman SURFONICs N-150) and branched isotridecyl ethoxylate (Huntsman SURFONICs TDA-9) (anionic)

Sulfonate surfactant such as alkyl benzene, alkyl naphthalene, alkyl indane and alkyl phenanthrene (anionic)

\section{Brief descriptions}

The core flooding experiments give some encouraging results $(15-75 \%)$ of enhanced oil recovery

These surfactants were used to form a copolymer via an inverse microemulsion system. This copolymer was used as a drilling fluid

Ultralow interfacial tensions were reached using these surfactants. It was also observed that addition of alkaline chemicals further reduces the interfacial tension between the oil and aqueous phases. Among these surfactants, SDBS performs better than the others

The double long-chain 1,3,5-triazine surfactants are very much active in reducing interfacial tension up to ultralow value. Therefore these surfactants have significant importance in the EOR method

These surfactants were used to prepare nanoemulsions. The prepared nanoemulsions show good efficiency in additional oil recovery from crude oil-saturated sand pack. The additional oil recovery was more than $30 \%$ OOIP

These gemini surfactants are effective in reducing interfacial tension between oil and water. These surfactants exhibit extraordinary aqueous stability even in high salinity and hard brines. At low concentrations the surfactants also show ultralow interfacial tension. The results from this study showed the potential of utilizing these surfactants at low concentrations and in harsh reservoir conditions

The newly developed surfactants are efficient for enhanced oil recovery as they can reduce the interfacial tension significantly. The Guerbet alkoxy carboxylate surfactants are alternatives to the sulfate surfactants for circumstances where the reservoir temperature is high and alkali is not included in the formulation

These surfactants are more soluble in $\mathrm{CO}_{2}$. So these surfactants can form stable $\mathrm{CO}_{2}$ foam easily which is a very appropriate candidate for EOR application for mobility control

All these surfactants are very active at low concentration. Synergistic effect was found when ethanolamine was used with the surfactants of microemulsions and are adsorbed at the interface rather than in the bulk phase. Surfactants are classified into four groups based on the charge of the head group such as anionic, cationic, nonionic and zwitterionic. Anionic surfactants such as sodium dodecylsulfate (SDS) are negatively charged in nature, but a small cation sodium ion occupies the counterpart. Anionic surfactants are most widely used in oil recovery process. Their adsorption phenomena in sandstone and carbonate are different. Their adsorption in sandstone is relatively lower than that of carbonate (Zhang et al. 2006). The adsorption can be reduced by use of sodium carbonate with the anionic surfactants. Cationic surfactants are positively charged and they are easily adsorbed in anionic surfaces of clay and 
sand. Therefore, they are not extensively used in oil recovery process for sandstone reservoirs. In recent investigation, it has been proved that cationic surfactants like cetyltrimethylammonium bromide (CTAB) performed better than anionic surfactants in altering the wettability of the carbonate rock to a more water wet (Salehi et al. 2008). Nonionic surfactants do not form ionic bonds, but the ether groups of the nonionic surfactants can form hydrogen bonding with water so that nonionic surfactants show surface properties (Myers 2006). Therefore, these surfactants introduce their polarity from having an oxygen-rich portion of the molecule at one end and a large organic portion at the other. Nonionic microemulsions also produce ultralow interfacial tensions and show high solubilization parameter (Verkruyse and Salter 1985; Iglauer et al. 2009). Another type of surfactant, i.e., zwitterionic may contain both positive and negative charges. Recently, Wang et al. (2010) have applied such type of surfactant in surfactantpolymer flooding for enhanced oil recovery and have obtained good consistent results.

The unique properties of microemulsions differentiate them from ordinary emulsion. For formulation of microemulsions, different alkanes with carbon number from $\mathrm{C}_{6}$ to $\mathrm{C}_{18}$ are generally used. The physicochemical properties of microemulsions depend on the alkane carbon number, nature of cosurfactant and types of surfactants. For ionic microemulsion preparation, cosurfactants are added along with surfactant (Healy and Reed 1977; Willhite et al. 1980), but some other researchers did not consider cosurfactant as a main component (Nelson and Pope 1978; Osterloh and Jante 1992). Microemulsions actually contain a cosurfactant such as a medium chain alcohol (viz. propan-2-ol, butanol, isoamyl alcohol etc.) in combination with a primary surfactant (Barakat et al. 1983; Lalanne-Cassou et al. 1983). Owing to the diphilic nature of surfactants, they distribute their head and tail parts to the corresponding polar and nonpolar solvents forming a monolayer film between them. This fact decreases the interfacial tension to an ultralow value, which results in the solubilization of the otherwise immiscible components. Cosurfactant, a second surfactant is generally added to the surfactant to prepare microemulsions due to its well-documented roles in various applications especially in EOR (Zhou and Rhue 2000; Richardson et al. 1997; Pattarino et al. 2000; Cavalli et al. 1996) such as to (1) prevent the formation of rigid structures such as gels, liquid crystals and precipitates; (2) alter the viscosity of the system; (3) reduce interfacial tension; (4) increase the fluidity of the interface and thereby increase the entropy of the system; (5) increase the mobility of the hydrocarbon tail and allow greater penetration of the oil into this region; (6) modify the hydrophilic-lipophilic balance (HLB) values of surfactants; and (7) influence the solubility properties of the aqueous and oleic phases due to its partitioning between the phases. Several attempts to substitute traditional cosurfactants with other components (Sagitani and Friberg 1980; Osborne et al. 1988; Comelles and Pascual 1997), for example nonionic surfactants, alkanoic acids, alkanediols, amines, aldehydes, ketones, butyl lactate and oleic acid, have been carried out from the viewpoint of suitable applications of microemulsions. The role of cosolvents and additives in the preparation of microemulsions has also been reported in literature (Kahlweit et al. 1985; Wormuth and Kaler 1987; Kim et al. 1988). Commonly, cationic, anionic and nonionic surfactants are used to form microemulsions. Physicochemical properties of the microemulsions are different for different surfactant systems. Brine is generally considered a pseudo component for microemulsion system taking in account water and salt as single phase.

Type and structure of microemulsion

Microemulsion structure has a key role in the different physicochemical parameters of the applied fields. The specific structures of the microemulsions have been extensively studied by many researchers (Azouz et al. 1992; Wadle et al. 1993; Maidment et al. 1997; Mo et al. 2000; Li et al. 2010). The three basic types of microemulsions are direct (oil dispersed in water, o/w), reversed (water dispersed in oil, w/o) and bicontinuous. Like multiple emulsion, sometimes multiple microemulsion are also possible. In this type, another layer is formed outside the $\mathrm{o} / \mathrm{w}$ or w/o microemulsions. The schematic diagram of the basic three types of microemulsions is shown in Fig. 2. Microemulsion structure depends on salinity, water content, cosurfactant concentration and surfactant concentration. At higher water content, the microemulsion would be a water-external system with oil solubilized in the cores of the micelles. Although the mixtures remain single phase and thermodynamically stable, the microemulsion structure changes through a series of intermediate states (Bourrel and Schechter 1988). The structures of these intermediate states are not well known. However, the solutions are thermodynamically stable and isotropic. Salinity also can reverse the structure of the microemulsion. As salinity increases, the direct microemulsion changes to reverse microemulsion. At low salinity, the system remains in water-external phase, but with increasing salinity the system separates into an oil-external microemulsion.

\section{Surfactant/microemulsion flooding}

During the past 40 years, it has been reported that many surfactant formulations for EOR generally form multiphase microemulsions (Chiang and Shah 1980; Cayias et al. 
Fig. 2 Microemulsion structures: a reverse microemulsion, $\mathbf{b}$ direct microemulsion and c bicontinuous microemulsion

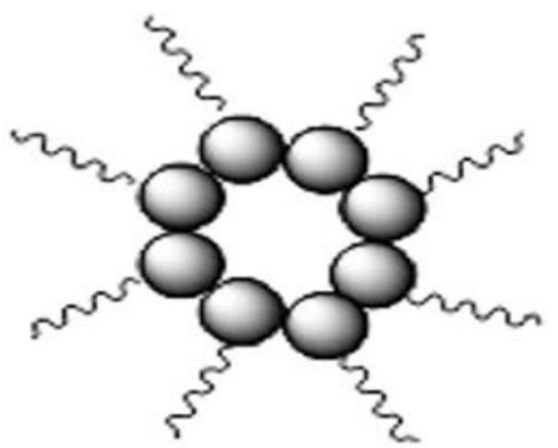

A

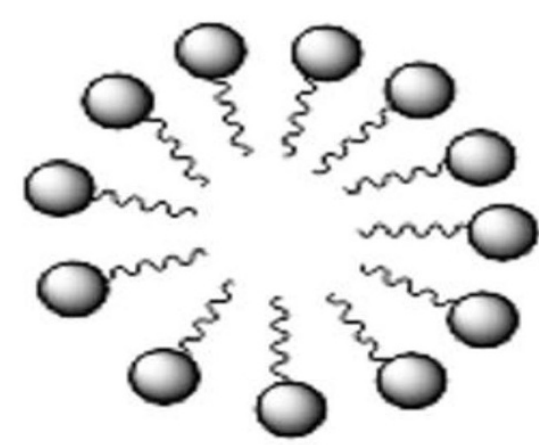

B

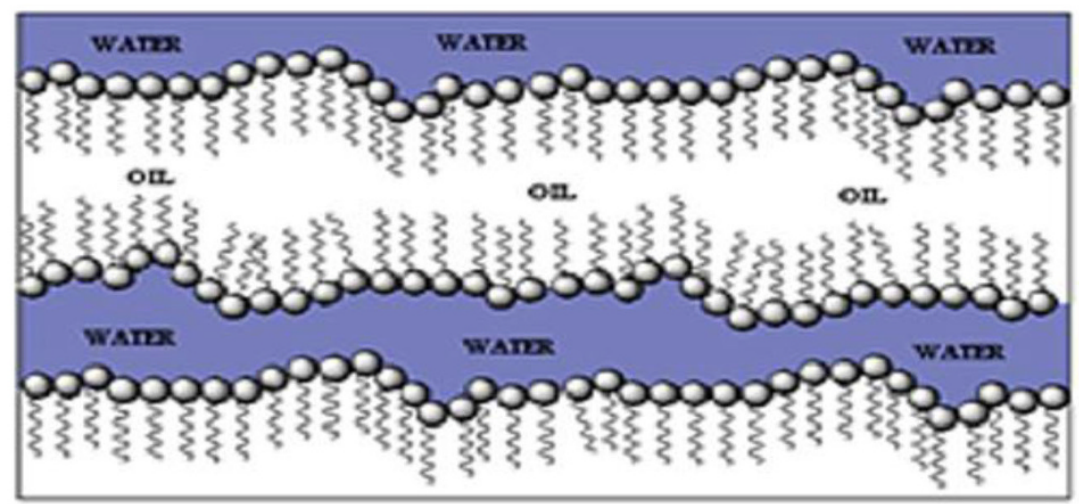

C

1977; Wilson et al. 1976; Schwuger et al. 1975). In chemical EOR methods, the process occurs with a certain degree of chemical interaction between the injected fluid and the reservoir fluid. This may be achieved by injecting polymer solutions, surfactant slugs, microemulsions or alkaline solutions. The main purpose of surfactant flooding is to reduce the interfacial tension between oil and water, thus increasing the displacement efficiency (Kwok et al. 1995). Surfactant solutions are not so efficient for better recovery of oil due to low viscosity compared to that of the oil. From this point of view, microemulsions are better replaceable injected fluids for their unique properties, which feature higher viscosity and induce low interfacial tension, increasing the oil extraction efficiency. Due to adsorption of surfactant molecules on the reservoir rock surfaces, low concentration of surfactant solutions are not allowable many times (Kassel 1989). Austad and Strand (1996) studied the microemulsion system and stated that very low interfacial tension may be reached with microemulsion systems. Under such circumstances, microemulsions flow more easily through the porous media, which enhances the oil extraction performance. For good efficiency of microemulsions and reasonable oil recovery efficiency, the surfactant must be chemically stable, reduce the interfacial tension between brine and crude oil and displace the oil without significant surfactant loss by adsorption on the reservoir rocks. Retention of surfactant is a most restrictive factor that affects the efficiency of the oil recovery process by microemulsion flooding (Glover et al. 1979). The microemulsion slug partitions into three phases such as a surfactant-rich middle-phase and surfactant-lean brine and oil phases (Healy and Reed 1974, 1977; Healy et al. 1976) in the intermediate salinity range. The surfactant-rich phase is the middle-phase microemulsion. In case of microemulsion flooding, a high concentration of surfactant must be used so as to form micelles that can solubilize or dissolve the reservoir oil. This phenomenon takes place via incorporation of small oil droplets in the micelle core, effectively causing miscibility in the system (Shindy et al. 1997).

\section{Mechanism of surfactant/microemulsion flooding}

In microemulsion flooding, the reservoir is flooded with water containing a small percentage of surfactant and other additives such as hydrocarbon, medium-chain alcohol and brine. The surfactant plays a key role in forming the exact type of microemulsion that reduces the interfacial tension of the target oil (Robert Moene, Shell Global Solutions). This is critical to both mobilize oil and enable it to escape from the rock. Generally speaking, whenever a waterflood has been successful, microemulsion flooding will be

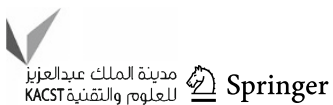


Fig. 3 Two-dimensional schematic diagram of microemulsion flooding

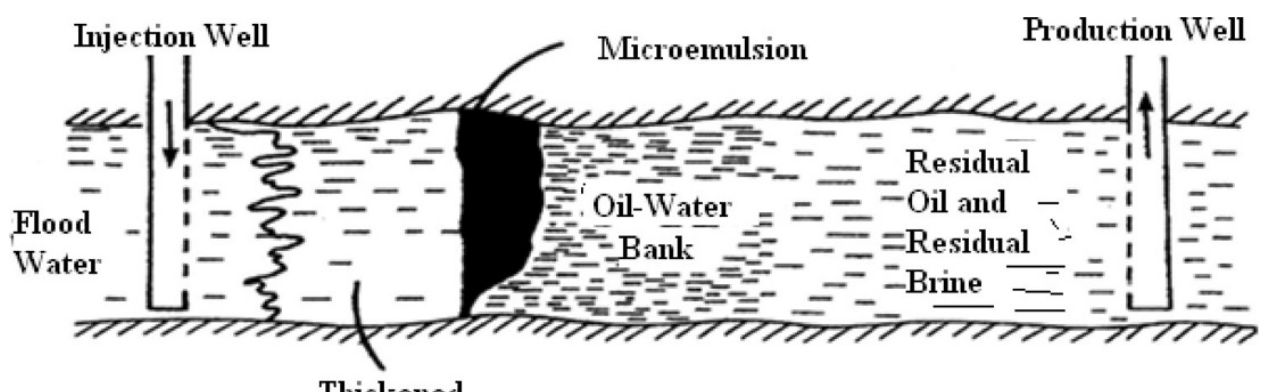

Thickened

Fresh Water applicable, while in many cases where flooding has failed owing to its poor mobility relationships, microemulsion flooding can still be successful mainly due to the required mobility control. A schematic diagram of surfactant/microemulsion flooding is shown in Fig. 3. The microemulsion flooding process is implemented as tertiary displacement near the end of a water flood. Figure 3 shows a tertiary process where residual oil saturation exists. A specified volume of surfactant slug $(0.5-1.0 \mathrm{PV})$ is injected. The micellar solution has a very low IFT with the residual oil and mobilizes the trapped oil, forming an oil bank ahead of the slug. The slug has a very low IFT with the brine and thus displaces brine as well as oil. Both oil and water flow in the oil bank. The thickened water is injected after the surfactant slug to drive the slug of microemulsion through the formation towards the production wells. A thickened water or mobility buffer slug consisting of a solution of polymer in water is used. The micellar solution must be designed in such a way that a favorable mobility ratio exists between the micellar slug and the oil bank. The viscosity of the micellar solution is adjusted to accomplish this. A polymer is often added to the micellar solution to increase its apparent viscosity. Thus, the process has the potential to increase both volumetric sweep efficiency and microscopic displacement efficiency. In some cases, a preflush is injected ahead of the micellar solution to adjust the brine salinity or $\mathrm{pH}$. The preflush solution may contain a sacrificial adsorbent that will be adsorbed on the rock and occupy adsorption sites. The purpose is to reduce adsorption and loss of the surfactant contained in the micellar solution.

Phase behavior of surfactant/cosurfactant-brine-oil system and optimization study

The phase behavior of surfactant-oil-brine system is the important key step in the laboratory to screen the proper surfactants for EOR. The microemulsion phase behavior changes from Winsor I to Winsor II through Winsor III with variation in salinity, temperature and pressure. Surfactant molecules in oil or in water form a variety of

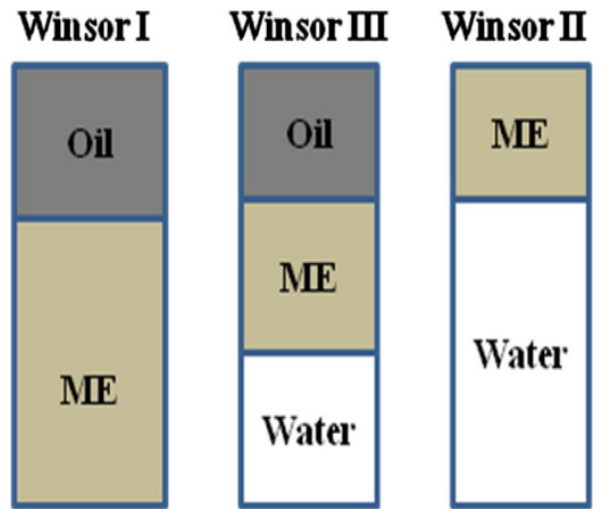

Fig. 4 Schematic diagram of Winsor-type phase behavior of microemulsion (ME)

structures when structure-assisted parameters such as water content, surfactant concentration, cosurfactant type, cosurfactant concentration, pressure and/or temperature are varied. Figure 4 shows the schematic diagram of the Winsor phase behavior of microemulsion, and Fig. 5 depicts the corresponding pseudoternary phase diagram. The middle-phase microemulsion consists of solubilized oil, brine, surfactant and alcohol. The lower to middle to upper phase transition of the microemulsion phase can be obtained by varying the following factors: (1) increasing salinity, (2) increasing alcohol concentration (propanol, butanol, pentanol and hexanol), (3) decreasing oil chain length, (4) changing temperature, (5) increasing total surfactant concentration, (6) increasing surfactant solution/oil ratio, (7) increasing brine/oil ratio and (8) increasing molecular weight of the surfactant.

Hussain et al. (1997) studied the three-phase microemulsion systems as a function of temperature and $\mathrm{pH}$. They showed that the presence of ethylene oxide (EO) moiety in the surfactant molecule made the surfactants less sensitive to salinity than an anionic surfactant. They also stated that the carboxylic ionic head group made the surfactant more stable to temperature than in simple EO nonionic surfactants. The phase behavior of $\mathrm{pH}$-dependent ether carboxylic acid system depends on salinity in the same way as in ethoxylated sulfonates (Qutubuddin et al. 1984). 
Fig. 5 Pseudoternary phase diagram of oil-water (brine)surfactant (cosurfactant) system (Winsor-type phase behavior of microemulsion where " $\phi$ " indicates the number of phases)

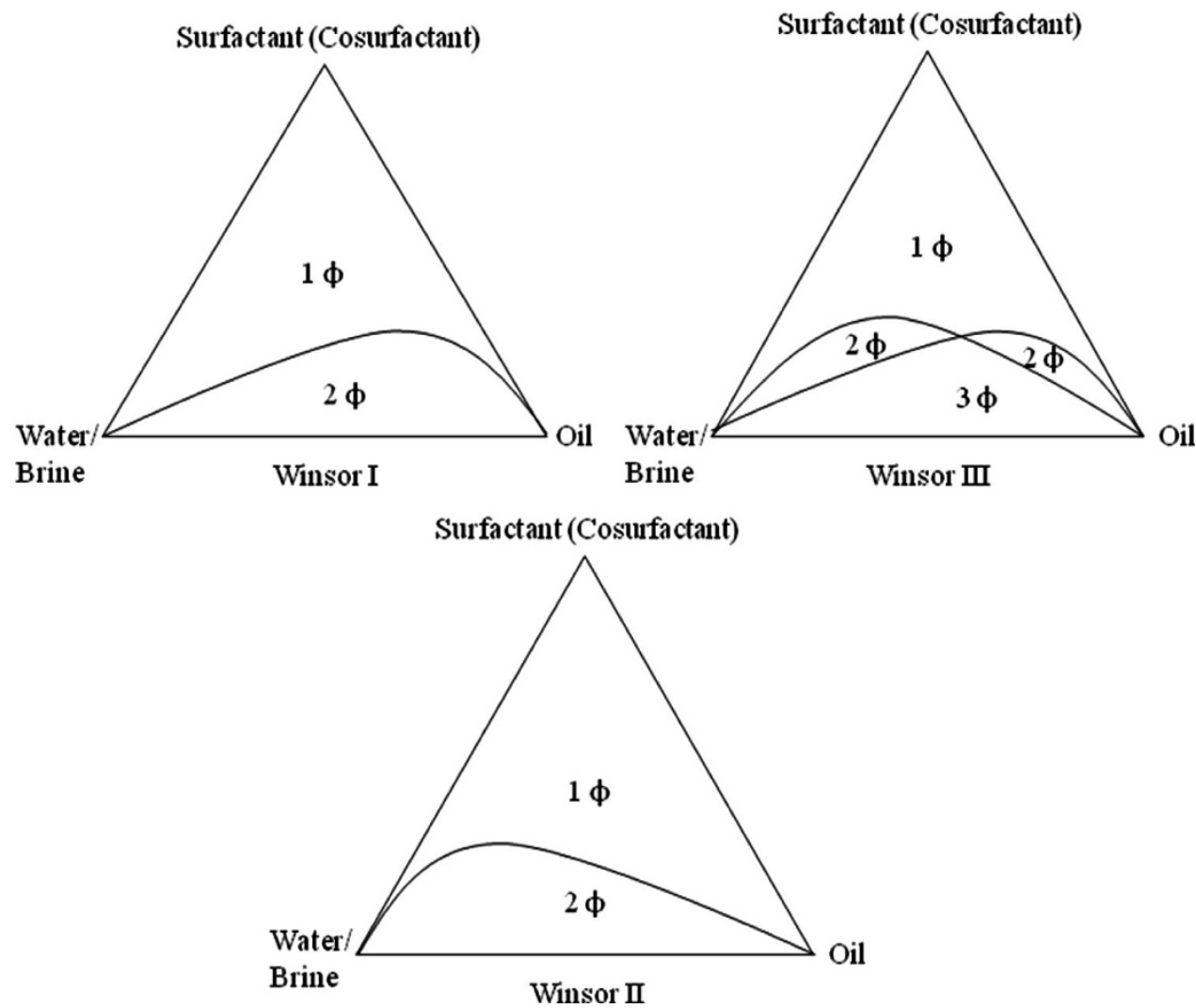

The middle-phase microemulsion is formed at low to high $\mathrm{pH}$ as a function of temperature at constant salinity. Increasing EO units in the surfactant molecules makes the surfactant molecule more hydrophilic, and hence high salinity and temperature are required to achieve the optimum region (Hussain et al. 1997). John and Rakshit (1994) studied the phase behavior and properties of cyclohexane/ $\mathrm{SDS} /$ propanol/water microemulsion system in the presence of $\mathrm{NaCl}$. They reported that the one-phase microemulsion region disappears completely at higher $\mathrm{NaCl}$ concentration. Abe et al. (1992) investigated the phase behavior and physiochemical properties of sodium octyl sulfate/ $n$-decane/1-hexanol/aqueous $\mathrm{AlCl}_{3}$ middle-phase microemulsion. They reported that at a particular salinity, a drastic change in the phase volume and physicochemical properties might be attributed to a phase inversion of $\mathrm{AlCl}_{3}$ middle-phase microemulsion from oil-rich to water-rich continuous phase with increasing $\mathrm{AlCl}_{3}$ concentration, which is quite a different behavior from that observed for monovalent and divalent salt systems. They also concluded that the nature of middle-phase microemulsion was very complicated and that its microstructure involves an intermicellar equilibrium incorporating various types of droplets, bicontinuous fluctuating and/or a rigid liquid crystal phase states, depending on the type of salt in the system. Bera et al. (2011) studied the interfacial tension and phase behavior of surfactantbrine-oil system for three nonionic surfactants. They reported that with increasing salinity, relative phase volume of middle-phase microemulsion would be increased due to increase conductivity of the solution and hydrophilicity of the molecules.

Solubilization capacity of microemulsion

High solubilization capacity of microemulsion depends on the formulation of microemulsion. Solubilization capacity of microemulsion is a function of surfactant category, oil type, cosurfactant nature, etc. Salts are also responsible for the variation of solubilization capacity of microemulsion. Microemulsions show a high level of solubilization capacity toward both oil and water simultaneously. This property makes them one of the most important tools in chemical EOR. In the presence of some additives, the water solubilization capacity of microemulsions is also influenced. Water solubilization of a microemulsion sometimes obeys the Bansal, Shah and O'Connell (BSO) equation. The equation suggests that maximum solubilization occurs if the summation of the carbon chain length of cosurfactant $\left(l_{\mathrm{cs}}\right)$ and oil $\left(l_{0}\right)$ is equal to the carbon chain length of the surfactant $\left(l_{s}\right)$, i.e., $\left(l_{\mathrm{cs}}+l_{0}=l_{s}\right)$. Recently in our few experimental studies, we also show the results of this rule (Bera et al. 2012c, 2014c).

Wei et al. (2011) reported that the water solubilization capacity increases initially with increasing ionic liquid 
(additive) concentration and reaches a maximum and then decreases with a further increase of ionic liquid concentration. They also reported the effect of the addition of salt to the microemulsion. The addition of salt diminishes the effective polar area of the surfactant by decreasing the thickness of the electrical double layer around the polar group. In this regard, packing parameter $(\mathrm{P})$ is defined as $v /$ $a l$, where $v$ is the effective volume of a surfactant molecule, $a$ is the effective area of its polar head and $l$ is the length of its hydrocarbon chain. As P increases, the water solubilization capacity of microemulsion decreases; because of the two counteracting factors, a maximum value of water solubilization capacity is observed. As the concentration of salt becomes higher, the latter effect plays a dominant role, which explains the further decrease in water solubilization capacity.

\section{Interfacial tension reduction}

Ultralow interfacial tension is required to recover the trapped oil with increasing capillary number. It is well known that ultralow interfacial tension plays an important role in oil recovery processes (Chiang and Shah 1980; Cayias et al. 1977; Wilson et al. 1976). The reasons of ultralow IFT's have been extensively investigated. The ultralow IFT are associated with phase behavior at plait point. At the plait point of liquid/liquid system, two phases become indistinguishable and IFT between the two equilibrium phases goes to zero. The microemulsion systems exhibit ultralow IFTs over wide ranges of salinities, surfactant concentrations and temperatures, suggesting that a critical phenomenon is involved. In 1977, Shah and Schechter (1977) demonstrated from their experimental results that there was direct correlation between interfacial tension and interfacial charge in various oil-water systems. Interfacial charge density is an important factor in lowering the IFT. Partition coefficient and IFT are strong functions of salinity. The minimum IFT occurs at the same salinity where the partition coefficient is observed to unity. Baviere (1976) proposed the same correlation between IFT and partition coefficient. Different factors (such as surfactant mixture ratio, salt concentration, temperature, pressure and oil types) influence the IFT.

Different category surfactants have different activities to reduce IFT. Sometimes, mixed surfactants show extra ability to reduce IFT. Therefore, the mixing ratio plays an important role. When interaction is not so strong, the two surface-active materials with equimolar concentration in the phase give the lowest IFT value among the different ratio mixtures (Rosen 1989; El-Batanoney et al. 1999).

The relative solubilities of surfactant in oil and water vary significantly with change in the salinity of the aqueous phase. At low salt concentration, most of the surfactant molecules stay in the aqueous phase, while at high salt concentration, the surfactant molecules preferentially dissolve into the oil phase. An equal distribution of surfactant in both oil and water phase is observed at a particular salinity, called optimal salinity, which produces the lowest IFT (Sayyouh 1994). The solubility of surfactant molecule in aqueous medium is reduced by salt (Anderson et al. 1976). However, at a certain concentration of surfactant, the presence of $\mathrm{NaCl}$ salt (up to a certain concentration) may promote the surfactant migration toward the interfacial layer from the bulk phase, generating a substantial decrease in the IFT between oil water (Schechter and Wade 1976; Bera et al. 2014a, b, c). Therefore, the IFT decreases with increasing salinity up to a certain salt concentration and then increases. This salt concentration is generally known as optimal salinity.

Viscosity and density of microemulsion

The magnitudes of the viscosity and density of displacing fluid relative to the displaced fluid are important design variables that affect volumetric displacement efficiency. The tendency for gravity override and underride to occur is determined by relative densities of the displaced and displacing fluid. Areal and vertical sweep efficiencies are in large measure determined by the mobility ratio in the displacement process. Both viscosity and density are functions of microemulsion composition. Viscosity, in particular, can be varied over a wide range by proper adjustment of composition and/or by polymer addition.

The viscosity of microemulsions depends on the structure of the microemulsion, i.e., whether it is water- or oilexternal. It is well known that at low water content, the system is oil-external, and at high water content, the system shows the reverse, i.e., water-external. The viscosity of the microemulsion increases as water content increases, creating swollen micelles. At the $50 \%$ water content, the viscosity of the microemulsion increased to two orders of the initial value. At higher water content, after inversion to a water-external system, the viscosity decreases with further addition of water. In general, the viscosity of displacing slug has been modified by addition of a polymer, such as polyacrylamide or biopolymer. The viscosity of the microemulsion can be modified by adding a cosurfactant (medium chain alcohol) and/or polymer to the microemulsion.

Applications of microemulsions in enhanced oil recovery

The use of microemulsion is of high interest in many aspects of crude oil exploitation in EOR, especially due to the ultralow interfacial tension values attained between the 
Fig. 6 Micellar-polymer flooding technique for enhanced oil recovery

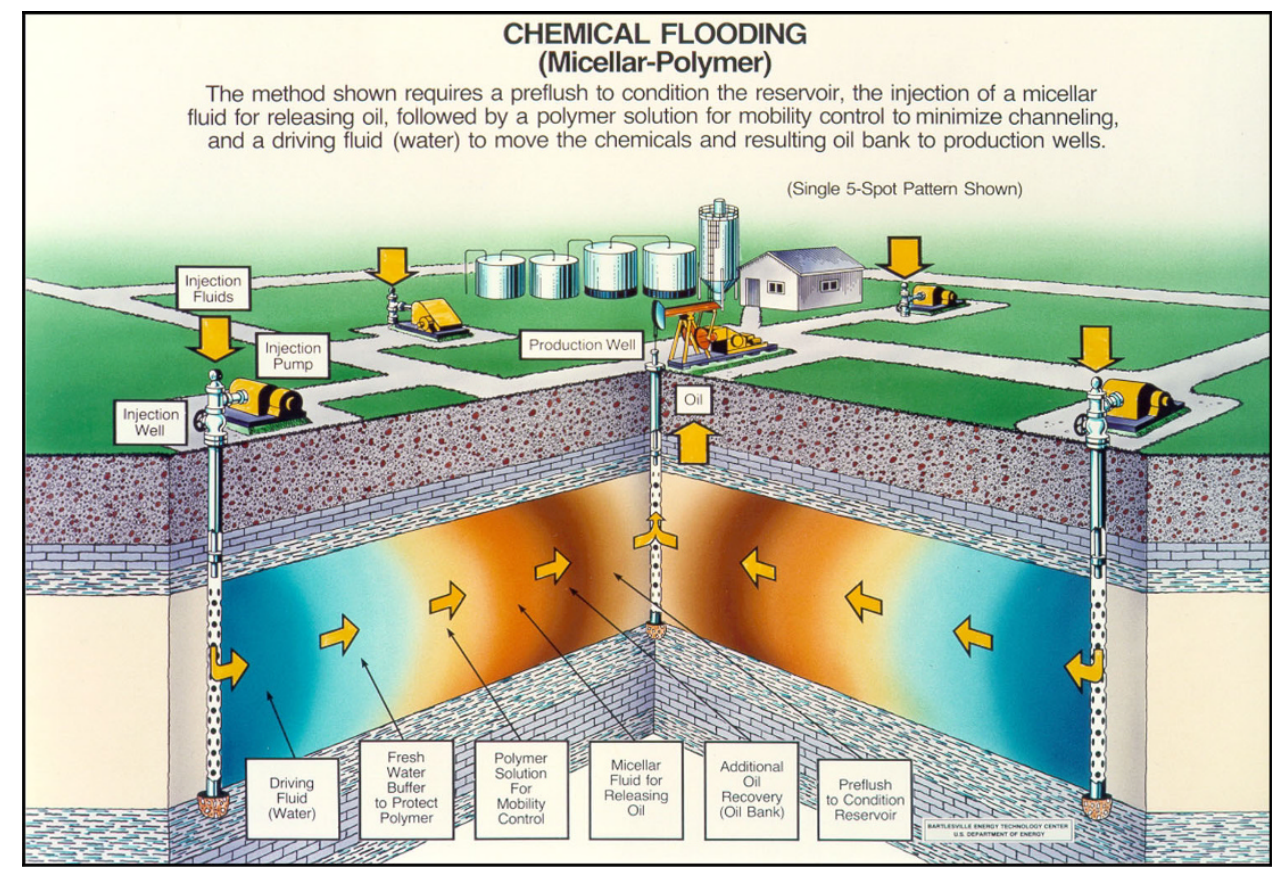

contacting oil and water phases. Microemulsion flooding in EOR can be applied over a wide range of reservoir conditions due to its exclusive ultralow interfacial tension property (Pottmann 1974; Santanna et al. 2009; Jeirani et al. 2013a). In cases where the pressure exerted by water on the oil phase is not able to overcome capillary forces sufficiently, microemulsions are the key to extracting more than just a minor portion of crude oil. Properly balanced microemulsions are able to do so by drastically reducing the interfacial tension to the magnitude of $0.001 \mathrm{mNm}^{-1}$. This is also known as chemical flooding. Figure 6 shows the chemical enhanced oil recovery by micellar-polymer flooding.

Healy and Reed (1974) reported on some fundamentals of microemulsion flooding, especially viscosity, interfacial tension and salinity, relating the results of phase behavior of self-assembled systems to the Winsor's concepts. Austad et al. (1994a, b) discussed the physicochemical aspects involved in this method, particularly the interactions existing within specific polymer-surfactant and microemulsion systems applied in EOR. In microemulsion techniques, the oil reservoir is flooded with water containing a small percentage of surfactant and other additives. This solution reacts with natural acids in the trapped oil, making a microemulsion similar to soap lather. As a result, lowering of interfacial tension between oil and water has been found. This is critical to both mobilizing oil and enabling it to escape from the rock. Microemulsion is injected into reservoirs in EOR processes and lowers the IFT to mobilize the residual oil left trapped in the reservoirs after water flooding (Engelskirchen et al. 2007;
Barnes et al. 2008; Santanna et al. 2009; Jeirani et al. 2013b). Tertiary oil recovery by means of microemulsions has been the main focus due to the ability to dissolve oil and water simultaneously in addition to attainment of very low interfacial tension of the system. Therefore, the design and analysis of chemical flooding processes for EOR depend on calculations of phase equilibria for these systems that are composed of water or brine, oil, surfactant and cosurfactant (usually a medium chain alcohol). Consequently, understanding the phase behavior of these systems is of fundamental importance to the development of any surfactant-based chemical flooding process. Microemulsions employed in EOR may be either oil-external (also called soluble oil) or water-external; mostly, they contain crude oil from the reservoir in which they are injected. The design of a microemulsion for a specific reservoir is basically a trial-and-error procedure; that is, the formulation of the microemulsion slug for a particular reservoir depends on the reservoir condition after the secondary recovery process and the properties of the microemulsion slug itself.

\section{Conclusions}

Microemulsions are very effective injecting fluids for extracting residual oil from reservoir in chemical EOR. Different chemical EOR techniques have been used for the last several decades in the oil recovery process. The drawbacks of the processes have forced to invent new techniques in EOR. Research in EOR is attributed to design 
and implementation of novel chemical methods. Mixtures, particularly of surface-active chemical substances, are incorporated in the injection formulations in this technology. This aims at oil displacement that takes place due to attaining ultralow interfacial tensions and reduced fluid viscosity in oil reservoirs. Beside this, microemulsions show extraordinary water solubilization capacity which again makes them capable for excellent injecting fluids in chemical EOR techniques. Laboratory investigations are now successful for enhanced oil recovery from sand pack or original cores. But this microemulsion slug has not been injected into the field to test its efficiency. Only few pilot tests have been implemented till now and these have been successful. So it is recommended that microemulsion flooding has proper efficiency to extract oil from natural reservoirs and can be used for pilot tests.

Acknowledgments The authors gratefully acknowledge the financial assistance provided by the Council of Scientific and Industrial Research (CSIR) (Project: 22(0649)/013/EMR-II), New Delhi, and the Department of Petroleum Engineering, Indian School of Mines, Dhanbad, India. Thanks are also extended to all individuals associated with the project.

Open Access This article is distributed under the terms of the Creative Commons Attribution License which permits any use, distribution, and reproduction in any medium, provided the original author(s) and the source are credited.

\section{References}

Abe M, Schechter RS, Selliah RD, Sheikh B, Wade WH (1987) Phase behavior of branched tail ethoxylated carboxylate surfactant/ electrolyte/alkane systems. J Dispers Sci Technol 8:157-172

Abe M, Yamazaki T, Ogino K (1992) Phase behavior and physicochemical properties of sodium octyl sulfate/ $n$-dodecan/1-hexanol/aqueous $\mathrm{AlCl}_{3}$ middle-phase microemulsion. Langmuir 8:833-837

Anderson DR, Biduer MS, Dravis MT, Manning CD, Schevan LE (1976) Interfacial tension and phase behaviour in surfactant brine oil systems. Presented at SPE Improved Oil Recovery Symposium, Tulsa, OK

Austad T, Strand S (1996) Chemical flooding of oil reservoirs-4. Effects of temperature and pressure on the middle phase solubilization parameters close to optimum flood conditions. Colloids Surf A 108:243-252

Austad T, Fjelde I, Veggeland K (1994a) Adsorption VI. Nonequilibrium adsorption of ethoxylated sulfonate onto reservoir cores in the presence of xanthan. J Pet Sci Eng 12:1-8

Austad T, Fjelde I, Veggeland K, Taugbol K (1994b) Physicochemical principles of low tension polymer flood. J Pet Sci Eng 10:255-269

Auvray L, Cotton JP, Ober R, Taupin C (1984) Structure and phase equilibria of microemulsions. J Phys 45:913-925

Azouz IB, Ober R, Nakache E, Williams CE (1992) A small angle $\mathrm{X}$-ray scattering investigation of the structure of a ternary waterin-oil microemulsion. Colloids Surf A 69:87-97

Bai Y, Xiong C, Shang X, Xin Y (2014) Experimental study on ethanolamine/surfactant flooding for enhanced oil recovery. Energy Fuels 28:1829-1837
Barakat Y, Fortney LN, Schechter RS, Wade WH, Yiv S, Graciaa A (1983) Criteria for structuring surfactants to maximize solubilization of oil and water: II. Alkyl benzene sodium sulfonates. J Colloid Interface Sci 92:561-574

Barnes JR, Smith JP, Smith JR, Shpakoff PG, Raney KH, Puerto MC (2008) Phase behavior methods for the evaluation of surfactants for chemical flooding at higher temperature reservoir conditions. Paper SPE 113314, presented at the 2008 SPE/DOE Improved Oil Recovery Symposium, Tulsa, OK

Baviere M (1976) Phase diagram optimization in micellar systems. Presented at the 51st annual fall technical conference and exhibition of the society of petroleum engineers of AIME, New Orleans, USA

Bera A, Ojha K, Mandal A, Kumar T (2011) Interfacial tension and phase behavior of surfactant-brine-oil system. Colloids Surf A 383:114-119

Bera A, Ojha K, Mandal A, Kumar T (2012a) Phase behavior and physicochemical properties of (sodium dodecyl sulfate + brine + propan-1-ol + heptane $)$ microemulsions. J Chem Eng Data 57:1000-1006

Bera A, Kumar S, Mandal A (2012b) Temperature-dependent phase behavior, particle size and conductivity of middle-phase microemulsions stabilized by ethoxylated nonionic surfactants. J Chem Eng Data 57:3617-3623

Bera A, Ojha K, Kumar T, Mandal A (2012c) Water solubilization capacity, interfacial compositions and thermodynamic parameters of anionic and cationic microemulsions. Colloids Surf A 404:70-77

Bera A, Kumar T, Ojha K, Mandal A (2014a) Screening of microemulsion properties for application in enhanced oil recovery. Fuel 121:198-207

Bera A, Mandal A, Guha BB (2014b) Effect of synergism of surfactant and salt mixture on interfacial tension reduction between crude oil and water in enhanced oil recovery. J Chem Eng Data 59:89-96

Bera A, Kumar T, Mandal A (2014c) Physicochemical characterization of anionic and cationic microemulsions: water solubilization, particle size distribution, surface tension and structural parameters. J Chem Eng Data 59:2490-2498

Bostich JM, Hsieh WC, Koepke JW (1984) Process for enhanced oil recovery employing petroleum sulfonate blends. Patent number US $4446036 \mathrm{~A}$

Bouabboune M, Hammouch N, Benhadid S (2006) Comparison between microemulsion and surfactant solution flooding efficiency for enhanced oil recovery in TinFouye oil Field. Paper 2006-058, presented at the Petroleum Society's 7th Canadian International Petroleum Conference and 57th Annual Technical Meeting, Calgary, Alberta, Canada

Bourrel M, Schechter RS (1988) Microemulsion and related systems. Marcel Dekker Inc., New York

Bumajdad A, Eastoe J (2004) Conductivity of mixed surfactant waterin-oil microemulsions. Phys Chem Chem Phys 6:1597-1602

Cavalli R, Marengo E, Caputo O, Ugazio E, Gasco MR (1996) The effect of alcohols with different structures on the formation of warm o/w microemulsions. J Dispers Sci Technol 17:717-734

Cayias JL, Schechter RS, Wade WH (1977) The utilization of petroleum sulfonates for producing low interfacial tensions between hydrocarbons and water. J Colloid Interface Sci 59:31-38

Chai JL, Zhao JR, Gao YH, Yang XD, Wu CJ (2007) Studies on the phase behavior of the microemulsions formed by sodium dodecyl sulfonate, sodium dodecyl sulfate and sodium dodecyl benzene sulfonate with a novel fishlike phase diagram. Colloids Surf A 302:31-35

Chiang MY, Shah DO (1980) The effect of alcohol on surfactant mass transfer across the oil/brine interface and related phenomena. 
Presented at the SPE 5th International Symposium on oilfield and Geothermal Chemistry, Stanford, CA

Chilingar GV, Yen TF (1983) Some notes on wettability and relative permeability of carbonate rocks: II. Energy Resour 7:67-75

Comelles F, Pascual A (1997) Microemulsions with butyl lactate as cosurfactant. J Dispers Sci Technol 18:161-175

Dosher TM, Wise FA (1976) Enhanced oil recovery potential: an estimate. J Petrol Technol 28:575-585

El-Batanoney M, Abdel-Moghny T, Ramzi M (1999) The effect of mixed surfactants on enhancing oil recovery. J Surf Deterg 2:201-205

Elraies KA, Tan IM, Awang M, Fathaddin MT (2010) A new approach to low-cost, high performance chemical flooding system. Presented at the SPE Production and Operation Conference and Exhibition, Tunis, Tunisia

Engelskirchen S, Elsner N, Sottmann T, Strey R (2007) Triacylglycerol microemulsions stabilized by alkyl ethoxylate surfactantsa basic study. Phase behavior, interfacial tension and microstructure. J Colloid Interface Sci 312:114-121

Flaaten AK, Nguyen QP, Zhang J, Mohammadi H, Pope GA (2010) Alkaline/surfactant/polymer chemical flooding without the need for soft water. Presented at the SPE Annual Technical Conference and Exhibition, Denver, Colorado, USA

Gao B, Sharma MM (2013) A family of alkyl sulfate gemini surfactants. 2. Water-oil interfacial tension reduction. J Colloid Interface Sci 407:375-381

Glover FDS, Santanna VC, Barros Neto EI (1979) Surfactant phase behavior and retention in porous media. Soc Pet Eng $\mathbf{J}$ 19:183-193

Gurgel A, Moura MCPA, Dantas TNC, Barros Neto EL, Dantas Neto AA (2008) A review on chemical flooding methods applied in enhanced oil recovery. Braz J Pet Gas 2:83-95

Healy RN, Reed RL (1974) Physicochemical aspects of microemulsion flooding. Soc Pet Eng 14:491-501

Healy RN, Reed RL (1977) Immiscible microemulsion flooding. Presented at the SPE 4th symposium on improved oil recovery, Tulsa, OK

Healy RN, Reed RL, Carpenter CW (1975) A laboratory study of microemulsion flooding. Soc Pet Eng J 259:87-100

Healy RN, Read RL, Stenmark DG (1976) Multiphase microemulsion system. Soc Pet Eng J 261:147-160

Holm LW (1971) Use of soluble oils for oil recovery. J Pet Technol 23:1475-1483

Hussain A, Luckham PF, Tadros TF (1997) Phase behavior of ph dependent microemulsions at high temperatures and high salinity. Oil Gas Sci Technol Rev IFP 52:228-231

Iglauer S, Wu Y, Shuler P, Tang Y, Goddard WA III (2009) Alkyl polyglycoside surfactant-alcohol co-solvent formulations for improved oil recovery. Colloids Surf A 339:48-59

Iglauer S, Wu Y, Shuler P, Tang Y, Goddard WA III (2010) New surfactant classes for enhanced oil recovery and their tertiary oil recovery potential. J Pet Sci Eng 71:23-29

Jeirani Z, Mohamed Jan B, Si Ali B, Noor IM, See CH, Saphanuchart W (2013a) Formulation, optimization and application of triglyceride microemulsion in enhanced oil recovery. Ind Crop Prod 43:6-14

Jeirani Z, Mohamed Jan B, Si Ali B, Noor IM, See CH, Saphanuchart W (2013b) Formulation and phase behavior study of a nonionic triglyceride microemulsion to increase hydrocarbon production. Ind Crop Prod 43:15-24

John AC, Rakshit AK (1994) Phase behavior and properties of a microemulsion in the presence of $\mathrm{NaCl}$. Langmuir 10:2084-2087

Kahlweit M, Strey R, Hasse D (1985) Effect of salt concentration on interfacial behavior in the surfactant system water $+n$ - octadecane + diethylene glycol monohexyl ether. J Phys Chem 89:163-167

Kahlweit M, Strey R, Busses G (1990) Microemulsions: a qualitative thermodynamic approach. J Phys Chem 94:3881-3894

Kassel DG (1989) Chemical flooding-status report. J Pet Sci Eng 2:81-101

Kayalia IH, Liub S, Miller CA (2010) Microemulsions containing mixtures of propoxylated sulfates with slightly branched hydrocarbon chains and cationic surfactants with short hydrophobes or PO chains. Colloids Surf A 354:246-251

Kim V, Hilfiker R, Eicke HF (1988) Excess adsorption of hydrocarbons on the oil/water interface in $\mathrm{H}_{2} \mathrm{O} / \mathrm{AOT} /$ oil microemulsions in the presence of additives. J Colloid Interface Sci 121:579-584

Kumar R, Mohanty KK (2010) ASP flooding of viscous oils. Presented at the SPE Annual Technical Conference and Exhibition, Florence, Italy

Kwok W, Hayes RE, Nasi-El-Din HA (1995) Modeling dynamic adsorption of an anionic surfactant on Berea sandstone with radial flow. Chem Eng Sci 50:768-783

Lalanne-Cassou C, Carmone I, Fortney LN, Samii A, Schechter RS, Wade WH, Weerasoooriya U, Weerasoooriya V, Yiv S (1983) Minimizing cosolvent requirements for microemulsion formed with binary surfactant mixtures. J Dispers Sci Technol 8:137-156

Leung R, Hou MJ, Monohar C, Shah DO, Chun PW (1985) Microand microemulsions.In: Shah DO (ed). Am Chem Soc, Washington, DC

Levitt DB, Jackson A, Heinson C, Britton LN, Malik T, Dwarakanath V, Pope GA (2006) Identification and evaluation of highperformance eor surfactants. SPE Reserv Eval Eng 12:243-253

Li X, He G, Zheng W, Xiao G (2010) Study on conductivity property and microstructure of TritonX-100/alkanol $/ n$-heptane/water microemulsion. Colloids Surf A 360:150-158

Lu J, Liyanage PJ, Solairaj S, Adkins S, Arachchilage GP, Kim DH, Britton C, Weerasooriya U, Pope GA (2014) New surfactant developments for chemical enhanced oil recovery. J Pet Sci Eng 120:94-101

Maerker JM, Gale WW (1992) Surfactant flood process design for Loudon. SPE Reserv Eng J 7:36-44

Maidment LJ, Chen V, Warr GG (1997) Effect of added cosurfactant on ternary microemulsion structure and dynamics. Colloids Surf A 129-130:311-319

Mandal A, Bera A, Ojha K, Kumar T (2012) Characterization of surfactant stabilized nanoemulsion and its use in enhanced oil recovery. Paper SPE 155406-MS, presented at International Oilfield Nanotechnology Conference and Exhibition, 12-14 June, Noordwijk, The Netherlands

Mclendon WJ, Koronaios P, Enick RM, Biesmans G, Salazar L, Miller A, Soong Y, McLendon T, Romanov V, Crandall D (2014) Assessment of CO2-soluble non-ionic surfactants for mobility reduction using mobility measurements and CT imaging. J Pet Sci Eng 119:196-209

Meyers KO, Salter SJ (1980) The effect of oil brine ratio on surfactant adsorption from microemulsions. Presented at the SPE 55th annual fall technical conference and exhibition, Dallas, Texas

Mo C, Zhong M, Zhong Q (2000) Investigation of structure and structural transition in microemulsion systems of sodium dodecyl sulfonate $/ n$-heptane $/ n$-butanol/water by cyclic voltammetric and electrical conductivity measurements. J Electroanal Chem 493:100-107

Myers D (2006) Surfactant science and technology, 3rd edn. Wiley, Hoboken

Nakamae M, Abe M, Ogino K (1990) The effects of alkyl chain lengths of sodium alkyl sulfates and $n$-alkanes on microemulsion formation. J Colloid Interface Sci 135:449-454 
Nelson RC, Pope GA (1978) Phase relationship in chemical flooding. Soc Pet Eng J 18:325-338

Osborne DW, Middleton CA, Rogers RL (1988) Alcohol-free microemulsions. J Dispers Sci Technol 9:415-423

Osterloh WT, Jante MJ (1992) Surfactant-polymer flooding with anionic $\mathrm{PO} / \mathrm{EO}$ surfactant microemulsions containing polyethylene glycol additives. Paper SPE 24151, presented at the SPE 8th Symposium on enhanced oil recovery, Tulsa, OK

Pattarino F, Marengo E, Trotta M, Gasco MR (2000) Combined use of lecithin and decvl polyglucoside in microemulsions: domain of existence and cosurfactant effect. J Dispers Sci Technol $21: 345-363$

Pottmann FH (1974) Secondary and tertiary oil recovery process. Interstate Oil Compact Commission, Oklahoma City

Purwono S, Murachman B (2001) Development of non petroleum base chemicals for improving oil recovery in Indonesia. Paper SPE 68768, presented at the SPE Asia Pacific Oil and Gas Conference and Exhibition, Jakarta, Indonesia

Qiao W, Li J, Zhu Y, Cai H (2012) Interfacial tension behavior of double long-chain 1, 3, 5-triazine surfactants for enhanced oil recovery. Fuel 96:220-225

Qutubuddin S, Millar CA, Fort T (1984) Phase behavior of pHdependent microemulsions. J Colloid Interface Sci 101:46-58

Richardson CJ, Aboofazeli A, Lawrence MJ, Barlow JD (1997) Prediction of phase behavior in microemulsion systems using artificial neural networks. J Colloid Interface Sci 187:296-303

Rosen MJ (1989) Selection of surfactant pairs for optimization of interfacial properties. J Am Oil Chem Soc 66:1840-1947

Sagitani H, Friberg SE (1980) Microemulsion systems with a nonionic cosurfactant. J Dispers Sci Technol 1:151-164

Salehi M, Johnson SJ, Liang JT (2008) Mechanistic study of wettability alteration using surfactants with applications in naturally fractured reservoirs. Langmuir 24:14099-14107

Santanna VC, Curbelo FDS, Castro Dantas TN, Dantas Neto AA, Albuquerque HS, Garnica AIC (2009) Microemulsion flooding for enhanced oil recovery. J Pet Sci Eng 66:117-120

Sayyouh MH (1994) Alkalines can improve miscibility of a surfactant-oil-brine system and areal sweep efficiency of oil. Oil Gas 1:13-17

Sayyouh MH, Abdel-Waly AA, George J, Salama AO (1991) Design of a microemulsion slug for maximizing tertiary oil recovery efficiency. SPE 20804

Schechter RS, Wade WH (1976) Research on tertiary oil recovery: annual report. University of Texas at Austin, Texas

Schramm LL, Stasiuk EN, Turner D (2003) The influence of interfacial tension in the recovery of bitumen by water-based conditioning and flotation of Athabasca oil sands. Fuel Proc Technol 80(2):101-118

Schulman JH, Stoeckenius W, Prince LM (1959) Mechanism of formation and structure of microemulsions by electron microscopy. J Phys Chem 63:1677-1680

Schwuger MJ, Stickdorn K, Plummer, MA, Roszelle, WO (1975) Patent number US3901317

Scriven LE (1976) Equilibrium bicontinuous structure. Nature 263:123-125

Shah DO (1981) Fundamental aspects of surfactant-polymer flooding process. Keynote paper presented at the European Symposium on Enhanced Oil Recovery, Bournemouth, England

Shah DO (ed) (1985) Macro- and microemulsions. ACS Symposium Series 272; American Chemical Society, Washington, DC

Shah DO, Schechter RS (1977) Improved oil recovery by surfactant and polymer flooding. Academic, New York
Sharma MK, Shah DO (1985) Macro- and microemulsion. In: Shah DO (ed). Am Chem Soc, Washington, DC

Shindy AM, Darwich TD, Sayyaouh MH, Abdel-Aziz O (1997) Development of an expert system for EOR method selection. Paper SPE-37708-MS, presented at the SPE Middle East Oil Show and Conference, 15-18 March, Bahrain

Southwick JG, Svec Y, Chilek G, Shahin GT (2010) The effect of live crude on alkaline-surfactant-polymer formulations: implications for final formulation design. SPE-135357-MS, presented at the SPE Annual Technical Conference and Exhibition, 19-22 September, Florence, Italy

Stoeckenius W, Schulman JH, Prince LM (1960) The structure of myelin figures and microemulsionsa s observed with the electron microscope. Kolloid-Z 169:170-178

Trabelsi S, Argillier J, Dalmazzone C, Hutin A, Bazin B, Langevin D (2011) Effect of added surfactants in an enhanced alkaline/heavy oil system. Energy Fuels 25:1681-1685

Verkruyse LA, Salter SJ (1985) Potential use of nonionic surfactants in micellar flooding. Paper SPE 13574, presented at the SPE International Symposium on Oilfield and Geothermal Chemistry, Phoenix, Arizona

Wadle A, Förster Th, von Rybinski W (1993) Influence of the microemulsion phase structure on the phase inversion temperature emulsification of polar oils. Colloids Surf A 76:51-57

Wan T, Yao J, Zishun S, Li W, Juan W (2011) Solution and drilling fluid properties of water soluble AM-AA-SSS copolymers by inverse microemulsion. J Pet Sci Eng 78:334-337

Wang D, Liu C, Wu W, Wang G (2010) Novel surfactants that attain ultra-low interfacial tension between oil and high salinity formation water without adding alkali, salts, cosurfactants, alcohol and solvents. Paper SPE 127452, presented at the SPE EOR Conference at Oil and Gas West Asia, Muscat, Oman

Wei J, Huang G, Yu H, An C (2011) Efficiency of single and mixed Gemini/conventional micelles on solubilization of phenanthrene. Chem Eng J 168:201-207

Willhite GP, Green DW, Okoye DM, Looney MD (1980) A study of oil displacement by microemulsion systems-mechanisms and phase behavior. Soc Pet Eng J 20:459-472

Wilson PM, Murphy CL, Foster WR (1976) The effects of sulfonate molecular weight and salt concentration on the interfacial tension of oil-brine-surfactant systems. Paper SPE 5812-MS, presented at the SPE Improved Oil Recovery Symposium, 22-24 March, Tulsa, OK

Winsor PA (1954) Solvent properties of amphiphile compounds. Butterworth's Scientific Publications, London

Wormuth KR, Kaler EW (1987) Amines as microemulsion cosurfactants. J Phys Chem 91:611-617

Yangming Z, Huanxin W, Zulin C, Qi C (2003) Compositional modification of crude oil during oil recovery. J Pet Sci Eng 38:1-11

Zhang DL, Lui S, Yan W, Puerto M, Hirasaki J, Miller CA (2006) Favorable attributes of alkali-surfactant-polymer flooding. Paper SPE 99744, presented at the SPE symposium on improved oil recovery, Tulsa, OK

Zhou M, Rhue RD (2000) Effect of interfacial alcohol concentrations on oil solubilization by sodium dodecyl sulfate micelles. J Colloid Interface Sci 228:18-23

Zhu Y, Xu G, Gong H, Wu D, Wang Y (2009) Production of ultra-low interfacial tension between crude oil and mixed brine solution of Triton X-100 and its oligomer Tyloxapol with cetyltrimethylammonium bromide induced by hydrolyzed polyacrylamide. Colloids Surf A 332:90-97 